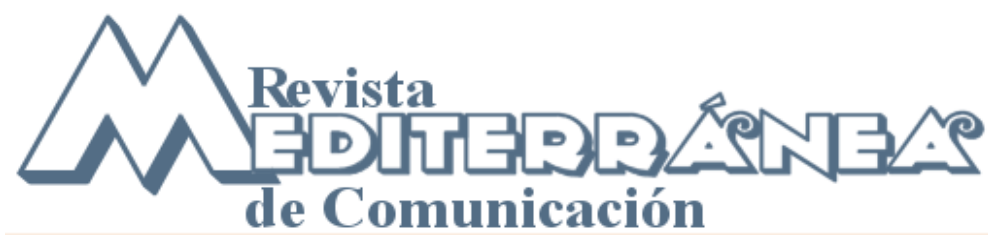

\title{
La base electoral de Obama, redes sociales virtuales y reales: los casos de generation engage y moms for Obama
}

\section{The electoral support of Obama, real and social virtual networks: the case of 'Generation Engage' and 'Moms for Obama'}

\author{
Prof. Dr. José Luis González \\ Universidad Miguel Hernández \\ Departamento de Estudios Económicos / Área de Periodismo \\ Campus de Elche (edificio Torreblanca) \\ Avenida de la Universidad $\mathrm{s} / \mathrm{h}$ \\ 03202 Elche (Alicante) \\ jose.gonzalez@umh.es \\ +34966 $658831 / 677567699$
}

\section{Resumen}

Después de un año de la victoria electoral de Barack Obama, el actual presidente de los Estados Unidos mantiene unas cotas de popularidad muy elevadas, máxime si tenemos en cuenta la actual coyuntura de crisis que afecta de manera frontal a los Estados Unidos. El presente artículo, a partir del estudio de un estado concreto: Carolina del Norte, explica cuáles son las bases sobre las que se sustenta dicho apoyo, bases que tienen que ver con un trabajo realizado desde las elecciones primarias del Partido Demócrata y que se sustentan en un adecuado uso de las redes sociales, tanto virtuales como reales. Para demostrar esta hipótesis, se muestran los resultados de un vasto trabajo de campo realizado, fundamentalmente con dos redes que han centrado sus esfuerzos en afianzar el voto joven y el voto femenino: 'Generation Engage' y 'Mom for Obama'.

\section{Palabras clave}

Política, Periodismo, Gobierno, Comunicación, Cibernética, Internet, Opinión Pública, Estados Unidos.

\section{Abstract}

After one year of the election victory of Barack Obama, the current president of the United Status, has a very high level of popularity, especially bearing in mind the current crisis that affects the United States. This article, from the study of a particular state: North Carolina, explains the keys of support, keys that are related to work done since the primaries and the Democratic Party behind in an appropriate use of social networks, both virtual and real. To prove this hypothesis, we show the results of an extensive field work carried out mainly with two networks that have focused their efforts on strengthening the youth vote and women voters: Generation Engage and 'Moms for Obama. 


\section{Key Words}

Political, Journalism, Government, Communication, Cibernética, Internet, Public Opinion, United States.

\section{Introducción}

Un estado como Carolina del Norte ha dado en bastantes ocasiones respaldo electoral al Partido Demócrata, pero tradicionalmente la conservadora sociedad de este territorio que hace de puente, en la costa del Atlántico, entre el sur y el norte, ha sido mayoritariamente republicana. Carolina del Norte es uno de los viejos estados fundacionales donde rige el actual estado democrático desde el año 1792; es decir, desde George Washington hasta la fecha, los vecinos de Carolina del Norte han tenido citas con las urnas de manera ininterrumpida. En el siglo XIX, Carolina del Norte dio su apoyo mayoritario a candidatos demócratas que luego se convertirían en presidentes como Andrew Jackson, Martin Van Buren, Franklin Pierce o el más conocido James Buchanan. Incluso en 1856, cuando la mayoría de los americanos votaron por el republicano Lincoln (uno de los modelos en que se mira Obama), en este estado se dio el voto a Breckinridge. Grove, otro demócrata, también tuvo por tres veces el apoyo del pueblo de Carolina del Norte. Ya en el siglo $\mathrm{XX}$, se mantuvo la tendencia de voto demócrata en cada cita con las presidenciales hasta el primer mandado de Nixon en 1968. Roosvelt, por cuatro veces (1932, 1936, 1940 y 1944), Truman, Kennedy o Lyndon Johnson fueron respaldados mayoritariamente en las urnas por los ciudadanos de este estado que singularmente, en el año 1952, votaron por Stevenson cuando Eisenhower arrasó en el resto del país.

Salvando los cuatro años de Jimmy Carter (1976-1980), la historia más reciente nos demuestra que en Carolina del Norte, la tendencia del voto en las presidenciales ha sido republicana, con victorias de Nixon, Ronald Reagan y los Bush. Incluso Clinton perdió en Carolina del Norte en las elecciones que derrotó a Bush padre, y también perdió en este estado cuando se presentó a la reelección cuatro años después, teniendo el respaldo mayoritario de todo el país, aunque no en Carolina del Norte que prefirió dar su apoyo al conservador Dole. Tampoco le fueron las cosas demasiado bien a Al Gore y Kerry en 2000 y 2004, perdiendo en ambos casos la candidatura demócrata frente a la republicana que lideraba George W. Bush. Siendo paradójico el caso de 2004 cuando el demócrata John Kerry se presentaba con John Edwards como candidato a la vicepresidencia. Edwards, en aquel momento, era un político de reconocido prestigio en Carolina del Norte, territorio de donde es natural y donde forjó una extraordinaria carrera profesional, como letrado, y política, como senador. A pesar de todo, el ticket Kerry-Edwards perdió ante BushCheney, pese a dominar con claridad los debates televisivos de aquella campaña; sobre este aspecto las profesoras Quintas Frofue destacan que "la fuerza de la televisión es tal que puede condicionar no sólo el proceso electoral, sino también las decisiones y acciones de un gobierno"1. El caso es que tuvieron que pasar, por tanto, 28 años, para que los demócratas ganaran el voto popular en unas elecciones presidenciales norteamericanas, y en la presente investigación se analizan las claves de dicha victoria, y la base sobre la que se sustenta dicho

\footnotetext{
${ }^{1}$ Quintas Froufe, Eva y Natalia (2010): “Cara a cara electoral televisado. Análisis audiovisual de los debates entre los candidatos a la Presidencia del Gobierno Española (2008)", en Miguel Hernández Communication Journal, 1, páginas 20 a 39. Universidad Miguel Hernández, UMH (Elche-Alicante). Recuperado el 10 de Enero de 2010 de: http://mhcj.es/2010/01/23/quintas-froufe
} 
respaldo a Obama, respaldo que una vez pasados los seis meses de su llegada a la Casa Blanca se sigue manteniendo.

\section{Metodología}

Para el desarrollo de esta investigación se realizó un primer trabajo de campo consistente en una doble inmersión de diez días en la sede del Partido Demócrata de Carolina del Norte, y en la redacción del News \& Observer, en su redacción de Raleigh (Carolina del Norte). Además de la mera observación participante de las rutinas comunicacionales en ambos escenarios, resultaron fundamentales las diversas entrevistas mantenidas diariamente con el editor online del periódico de Raleigh, Dan Barkin, así como con el presidente del NCDP, también en la ciudad de Raleigh, Jerry Meek, así como con otros expertos El trabajo de campo se completó con entrevistas a responsables de la organización no gubernamental Generation Engage (Generación Comprometida), a profesores de la Universidad Estatal de Carolina del Norte (NCSU), así como con la realización de encuestas a estudiantes de postgrado de la citada Universidad, la más populosa del estado.

De igual modo, la investigación también se ha sustentado en la consulta bibliográfica de autores que están avanzando conclusiones relevantes referidas a la influencia de las redes sociales y los nuevos medios de comunicación online, etc. Siendo evidente que aún existiendo una bibliografía incipiente sobre todas estas cuestiones referidas a un plano nacional o internacional, no la hay tanto sobre casos locales, como el que nos ocupa, siendo estos ámbitos de la comunicación política un campo de enorme interés para el estudio. El estilo de la narración es el de la literatura científica, razonando y explicando los hallazgos obtenidos, aportando respuestas, comparando modelos y llegando a las pertinentes conclusiones.

\section{Vuelco electoral en el estado de Carolina del Norte}

¿Qué pasó el 4 de noviembre de 2008 en el estado de Carolina del Norte? Básicamente la respuesta en forma de titular periodístico sería que ocurrió un vuelco histórico. Barack Obama representante del Partido Demócrata obtuvo en este estado 2.123.334 votos que representaron el $49,9 \%$ del electorado y que le permitieron ganar los 15 'electoral votes' que aporta Carolina del Norte al conjunto del país; mientras que John McCain, representante del Partido Republicano, tuvo el respaldo de 2.109.281 votantes, es decir, el 49,5\% de los votos. El liberal Bob Barr fue votado por 25.421 personas, apenas un $0,6 \%$ del censo. Mirando a 2004 vemos con mayor perspectiva lo de vuelco histórico, ya que por aquel entonces Kerry se quedó en este estado en un $43,6 \%$ de los votos por un $56 \%$ que logró George W. Bush; es resumen, la subida de los demócratas fue del $6,3 \%$ y el descenso de los republicanos del 6,5\%, es decir, la diferencia entre ambas formaciones respecto a la situación de 2004 se fue hasta casi los 13 puntos, algo inédito en el territorio objeto de esta investigación.

Pero además de las presidenciales, los habitantes de Carolina del Norte tenían ese mismo día más citas con las urnas. Por ejemplo, para elegir su representante al Senado, resultando elegida la demócrata Kay Hagan, con 2.225.594 votos, un 52,7\%, muy por encima de Elizabeth Dole, del Partido Republicano que aspiraba a la reelección y no pasó del $44,2 \%$, con 1.868 .240 votos. El 
tercero en discordia fue un hombre, Christopher Cole, de los liberales, que apenas tuvo el respaldo del $3 \%$ del electorado. Pero sin lugar a dudas al tercer foco de atención de la política de Carolina del Norte en aquel momento se centraba en la gobernación del Estado, donde otra mujer y otra demócrata, Bev Perdue, derrotó al republicano Pat McCrory. Perdue fue votada por 2.120.847 personas, prácticamente el mismo número que votó a Obama, un $50,2 \%$, mientras que McCrory se quedó en 1.980 .734 votos, un 46,9\%. Así las cosas, los demócratas lograban un 'triplete' inédito en este estado, con victoria en las presidenciales, en el Senado y eligiendo a Beverly Perdue como gobernadora de Carolina del Norte.

¿Dónde se fraguó la victoria de los demócratas? Básicamente en las zonas más urbanas y universitarias, caso de Greensboro, Charlotte, Fayetteville o Raleigh, destacando dentro de los condados que circundan a la capital del estado (Raleigh) ciudades tradicionalmente viveros del voto demócrata como Chapel Hill. Por el contrario, el voto republicano tuvo su caldo de cultivo en todas las zonas más rurales del estado, algo similar a lo que ocurrió en el conjunto del país. Destaca en este sentido como los republicanos arrasaron en el denominado cinturón de la biblia: Watauga (oeste Carolina del Norte), la zona del oeste que toca con Tennesse y el resto pro Apalachia. Solamente los demócratas arañaron votos en el cinturón de la Biblia en Boone, también zona de influencia universitaria al estar allí ubicada la Appalachian State University. En estados como Carolina del Norte se puede ver muy claramente la existencia de dos américas: la urbana (universitaria) y la rural (tradicional y muy religiosa)

¿Por qué se produjo el vuelco en Carolina del Norte? Este artículo pretende insistir en la clave de unir adecuadamente estrategias reales y virtuales que tienen el mismo objetivo: acercar la política y al político a la ciudadanía. La candidatura de Obama, desde las primarias demócratas, supo aunar con certeza la estrategia puerta a puerta, veremos dos ejemplos posteriormente: Generation Engage y Moms for Obama, con un despliegue sin precedentes en la Red. El presidente del Partido Demócrata en Carolina del Norte, el abogado Jerry Meek, en exclusiva para esta investigación, explicaba que: "Las elecciones de noviembre de 2008 han sido históricas en Carolina del Norte. Obama ayudó para que se ganaran otras votaciones, es el caso de la gobernación del Estado o el senado, pero desde luego fue una gran noticia para nosotros que una mujer fuese elegida como Gobernadora del Estado, la primera vez en la historia. Y detrás de este éxito hubo un trabajo muy bien diseñado que consiguió implicar a miles de voluntarios en todo el estado". Miles de esos voluntarios fueron jóvenes universitarios, el voto joven resultó decisivo y así lo destaca Jerry Meek: "Conseguimos movilizar el voto joven, el voto universitario porque sabíamos que podía ser decisivo. Un $70 \%$ de los jóvenes con derecho a voto lo ejercieron, y esa es una cifra muy ilusionante. Además una amplia mayoría de esos jóvenes votantes, en Carolina del Norte, respaldaron las candidaturas demócratas de Obama, Hagan y Bev. Perdue" 2 . El número de voluntarios que trabajó para el Partido Demócrata de Carolina del Norte desde las primarias hasta las presidenciales fue de 27.000: "Esto también es histórico -dice Jerry Meekporque nunca se había conseguido movilizar a tantos voluntarios, muchísimos jóvenes han ido casa por casa, hablando con la gente, explicando porque se debía producir un cambio en la política norteamericana, su implicación ha sido increíble y clave en la victoria".

Esa idea de cercanía, de política hiperlocal, muy pegada al territorio, también la destaca el profesor Luis Olivieri como una de las grandes fortalezas de Obama y del conjunto del Partido Demócrata en las últimas elecciones. Olivieri, de origen latino, es profesor de North Carolina State

\footnotetext{
${ }^{2}$ Meek, Jerry, entrevista personal con el presidente del Partido Demócrata en Carolina del Norte, Raleigh (EE. UU), noviembre 2008
} 
University (NCSU) y especialista en política americana. Este experto resume la victoria de Obama en las siguientes cualidades: juventud, energía, multiculturalismo, imagen de cambio, facilidad para conectarse con la gente y capacidad para llegar a las comunidades y vecindarios. En referencia a alguna de estas cuestiones y centrando la cuestión en los resultados que se obtuvieron en Carolina del Norte, Olivieri explica algo que ha pasado muchas veces en comunidades autónomas españolas como Castilla-La Mancha: "Carolina del Norte ha sido mayoritariamente demócrata para la gobernación, pero tradicionalmente republicana para la presidencia. Ha habido durante las últimas décadas un sector demócrata que es conservador en ciertos asuntos y temas que podía respaldar a un candidato demócrata a gobernar el estado, pero que en las presidenciales votaba a Bush. Pero todo cambió el 4N porque la participación fue muy alta, las minorías votaron como nunca, también los jóvenes y las mujeres, y se produjo un cambio inesperado en un estado tan conservador como éste que optó por Obama y también por una demócrata para el Senado y una mujer, también demócrata, para gobernar el estado, algo sin precedentes en esta zona del país"3. En referencia a la clave del multiculturalismo, en el estado objeto de estudio fue importante, pero no tanto como en otros estados; así, en Carolina del Norte solamente un $2 \%$ del electorado latino se registró para votar. A pesar de este dato tan significativo, los dos entrevistados, tanto Jerry Meek, como Luis Olivieri, destacan el hecho que en Carolina del Norte el voto latino siga yendo para los demócratas, y la necesidad de "cuidar" ese nicho de votos porque el aumento de la población latina en el estado está siendo espectacular y calculan que las próximas elecciones presidenciales se llegará al 10\%. En relación a la minoría negra de Carolina del Norte, el profesor Olivieri considera que sí hubo una movilización sin precedentes de un colectivo con una habitual tendencia abstencionista. "La minoría negra del estado se registró para votar en las primarias y luego para las presidenciales, gobernación del estado y senado. Desde hace muchos años no habían votado tantos negros como en esta ocasión y su respaldo fue vital para el vuelco electoral", explica el profesor Olivieri. En este sentido, el profesor de NCSU, destaca otra clave como es la semejanza entre Obama y Martin Luther King: "Obama es un demócrata de centro-izquierda o moderado liberal, pero creo que por el momento no se le puede comparar completamente con el reverendo Martín Luther King, ni en sus acciones, ni en sus sacrificios. Sus ideas son muy parecidas, pero Obama no es un profeta, y Luther King sí lo fue". Sea como fuere la realidad demográfica de Carolina del Norte es que efectivamente el electorado latino sigue siendo pequeño en número, pero se están produciendo desde hace diez años migraciones internas desde el noreste del país de población latina y afroamericana que está cambiando progresivamente el perfil tradicional de este estado.

Otro entrevistado para esta investigación, el periodista del News \& Observer, Dan Barkin insiste en esta misma idea de la movilización electoral de colectivos como estudiantes, mujeres, negros, latinos, etc, pero destacando además que "los demócratas consiguieron llegar al voto blanco, de centro que, al fin y a la postre es quien decide las elecciones en estados como Carolina del Norte"4. Mientras, el profesor Enrique Pastor Seller profundiza en otra idea clave para el sentido final del presente ensayo: la de evitar la no participación, a partir de estrategias muy locales, algo que hicieron bien los demócratas en los comicios del 4 de noviembre de 2008 en Estados Unidos: "Generar estrategias relacionales de mayor impacto en las políticas sociales locales, clarificando objetivos y funciones, ofreciendo contenido real, motivación a participar, en definitiva, innovando en procesos y metodologías que eviten la inercia de la no participación y el acaparamiento del poder, facilitando accesos, compatibilizando participación asociativa y directa, gestión y

\footnotetext{
${ }^{3}$ Olivieri, Luis, entrevista personal con el politólogo de North Carolina State University, Raleigh (EE. UU), noviembre 2008

${ }^{4}$ Barkin, Dan, entrevista personal con el editor online del News \& Observer, Raleigh (EE.UU), noviembre 2008
} 
democratización"5. Moms for Obama o Generation Engage representan esa participación asociativa directa en el ámbito local, con el fin de movilizar al electorado a través de redes locales 'reales'.

¿Por qué se producen los vuelcos electorales? Pues en gran medida por una movilización del electorado joven al que erróneamente muchas veces se le considera dormido. El electorado joven resultó decisivo en la primera victoria de Zapatero en España, en 2004, y en noviembre de 2008 ocurrió algo similar en Estados Unidos con Obama. La revista Temas, en su no 176, correspondiente al mes de julio de 2009 aborda de manera monográfica el tema de la juventud y la participación política, y en su editorial parte de la base que en las actuales generaciones de jóvenes confluyen una serie de circunstancias socio-laborales y culturales que pueden abrir paso a una mayor implicación y participación política: “(...) Es necesario impulsar entre los jóvenes un gran debate ampliamente participativo, cuyo objetivo sea suscitar un mayor interés por lo colectivo. Un debate que debe abordarse desde las posibilidades que ofrecen las nuevas tecnologías, en la dirección de una mejora cualitativa de la democracia interna de los partidos, un retorno del debate ideológico como elemento enriquecedor, la apertura de nuevos cauces de implicación social, los avances en participación, la dignificación de la política y la lucha contra los comportamientos irregulares y éticamente criticables" ${ }^{\prime \prime}$.

\section{La Política 2.0 de Barack Obama}

La recuperación del voto joven y el voto femenino con estrategias puerta a puerta, así como el empleo inteligente de las nuevas tecnologías, introduciéndose con fuerza el empleo de las redes sociales resultaron fundamentales para el triunfo demócrata en Estados Unidos y en Carolina del Norte. El analista Juan Freire explica que tal circunstancia se fue consolidando desde las primarias demócratas: "Internet si tuvo mucho que ver en el resultado de las primarias en el Partido Demócrata. En el caso republicano, sólo Ron Paul se diferenció del resto gracias a su uso de las herramientas y redes digitales, pero aún así su éxito fue muy limitado, posiblemente por la marginalidad de sus propuestas. La presencia de Obama en la Red, desde las Primarias hasta su llegada a la Casa Blanca, no es más que una extensión y amplificación de su estilo de comunicación y relación con sus simpatizantes y votantes. Obama presentó un perfil muy diferenciado al que hasta ese momento había sido habitual en la política norteamericana"7.

El despligue y estrategia del equipo de Obama en Internet se focalizó en sitios como BarackObama.com, su perfil de Facebook, Twitter y MySpace: MyBarackObama.com, los canales de vídeo de You Tube y el propio Barack TV, dentro del portal BarackObama.com, el sitio WAP Obama Mobile, en la wikipedia, y en el blog BarackObama.com. El periodista Joe Raspers, director de New Media de la campaña de Obama, trabajó especialmente la wikipedia, respaldado por Chris Hughes (uno de los fundadores de Facebook). Para el blog, el presidente contó con Sam Graham Felsen, como bloguera jefe, responsable de un numeroso equipo de blogueros profesionales que trabajaron en la generación de contenidos y, fundamentalente, en la conversación con el ciudadano a través de los blogs. Kate Albraigh-Hanna fue la directora del

\footnotetext{
5 Pastor Seller, E, 'Participación ciudadana y política social', Ed. Azacanes, Praxis 13, marzo de 2009 , Toledo, pgs. 110-127

6 Editorial, 'Juventud y participación política', Fundación Sistema, Temas para Debate 176, julio de 2009, Madrid, pgs. 3-4

7 Freire, J, 'Internet en la campaña electoral americana', Soitu, noviembre de 2008, Madrid, disponible en: http://www.soitu.es/soitu/2008/10/02/pieldigital/1222965797_622282.htm
} 
equipo de vídeo de Obama para el blog, mientras que Scott Goodstein, ex asesor de Bill Clinton se responsabilizó de la estrategia SMS. Sobre la incursión del equipo de Obama en la estrategia del SMS, y en particular sobre la apuesta que hicieron por el iPhone, Juan Varela destacaba que: "Barack Obama, el presidente multimedia, ya está en el iPhone con toda la red social de MyBarackObama.com, y no es de extrañar, los demócratas usan los teléfonos móviles para acceder a Internet y los mensajes SMS mucho más que los republicanos"8. En este sentido, es reseñable el hecho que Obama iniciase esta estrategia a lo grande, enviando un SMS con su elección de Joe Biden como ticket a la vicepresidencia a 2,9 millones de teléfonos móviles, gracias a lo cual su campaña consiguió la mayor base de datos de móviles, jamás conocida, de usuarios interesados en política. Esa base de datos fue creciendo exponencialmente hasta el mismo día de las elecciones. El resumen de la importancia que el equipo de Obama dio a las nuevas herramientas de comunicación política (Política 2.0) son los casi 25 millones de usuarios que siguieron los sitios relacionados con Obama, mientras que McCain apenas atrajo a 3,5 millones de usuarios. 84 millones de posibles búsquedas en Google dan una idea de las simbiosis conseguidas entre Obama y la Política 2.0 (aplicación de blogs y redes sociales al ámbito de la política).

El nuevo ecosistema de medios se sostiene en tres patas: web 2.0, blogs y periodismo cívico. Los medios y los periodistas trabajan en un doble nivel con las comunidades y los blogueros, por una parte como filtradores de noticias y por otra como receptores de ideas de historias. Dichas comunidades y blogs son fuentes con los que hay que mantener una conversación constante, de lo contrario este ecosistema de medios perece. Se trata, en definitiva, de desarrollar una comunicación punto a punto o de red social, y la política 2.0 no es otra cosa que aplicar estas teorías al ámbito de la política. El equipo de Obama y sus redes de voluntarios creyeron en este nuevo ecosistema, aplicándolo hasta sus últimas consecuencias. "Desde un principio, el equipo de Obama tuvo claro que para llegar a los jóvenes había que ir donde están los jóvenes y los jóvenes están en Internet, están en Facebook, están en My Space, están en otras redes sociales, en blogs, foros, etc. Obama ha creído en la Red. Ha sido la primera vez que un político se toma verdaderamente en serio la ciberpolítica y la primera vez que ha dado unos resultados evidentes", asegura el presidente del Partido Demócrata en Carolina del Norte, Jerry Meek ${ }^{9}$. El politólogo Luis Olivieri coincide en el planteamiento de Meek: "La apuesta de Obama por la Política 2.0 ha sido vital para el intercambio de ideas y detalles en tiempo real. Vivimos en una época de fuerte carga audiovisual, y las candidaturas y electores han de usarlo masivamente para el flujo de ideas, opiniones, versiones y decisiones. Obama lo sabía y desde las primarias supo utilizar bien estas herramientas de la nueva política del siglo XXI"10. En definitiva todo pasa por la conversación, "el periodismo ciudadano bien entendido exige una redacción capaz de conversar en un plano de igualdad con sus usuarios y mantener así el máximo nivel de interactividad posible" ${ }^{11}$. Los medios convencionales, no en el grado deseado, también están intentando profundizar en esa conversación, un ejemplo estudiado es el de los espacios de participación del periódico líder de Carolina del Norte, el News \& Observer que tiene una de las ediciones online más potentes del grupo McClatchy. El periódico objeto de estudio coincidió con los días previos a las elecciones presidenciales, incluyendo la jornada correspondiente al último mitin que Obama dio en Raleigh. Uno de los espacios de participación más destacados es la blog zone donde se pueden encontrar

\footnotetext{
8 Varela, J, 'Con Obama en el iPhone', Soitu, noviembre de 2008, Madrid, disponible en: http://soitu.es/soitu/2008/10/04/sociedadcableada/1223105526_450020.html

${ }^{9}$ Meek, Jerry, entrevista personal con el presidente del Partido Demócrata en Carolina del Norte, Raleigh (EE. UU), noviembre 2008

${ }^{10}$ Olivieri, Luis, entrevista personal con el politólogo de North Carolina State University, Raleigh (EE. UU), noviembre 2008

${ }_{11}^{11}$ Martínez Mahugo, S, 'Estrategias y modelo de negocio en el periodismo ciudadano: el caso de Bottup', Congreso Internacional Blogs y Periodismo Cívico, 2009, Bilbao
} 
blogs como Under the Dome, todo un referente en política, liderado por el prestigioso periodista Ryan Tague, y en el que trabajan y colaboran hasta ocho periodistas profesionales, en constante diálogo con el usuario-ciudadano: "Algunos de los periodistas de este equipo tienen la labor de dinamizar las conversaciones, contestar a los ciudadanos, plantear debates, crear una discusión razonada e inteligente que sirva para fidelizar lectores que ocasiones pueden acabar convirtiéndose en fuentes"12. Espacios como el referenciado se convirtieron durante los meses previos a las elecciones presidenciales de 2008 en espacios de debate político seguidos por miles de ciudadanos, muchos de ellos jóvenes y muchos de ellos ciberactivistas.

\subsection{El ciberactivismo de 'Generation Engage'}

Un blog no es un medio, pero el conjunto de blogs sí que lo es: la blogosfera. Se trata de un medio de comunicación que puede cambiar la agenda pública, elevando al debate social cuestiones que los medios tradicionales no abordan o filtran. Este medio es cada vez más importante en el ámbito local: ciberperiodismo de proximidad. ¿Qué es el ciberactivismo?: "Es una estrategia para formar coaliciones temporales de personas que, utilizando herramientas en Red, generen la masa crítica de información y debate para que trascienda la blogosfera y salga a la calle o se modifique de una manera susceptible el comportamiento de un número de personas"13.

Generation Engage parte de esta estrategia de la que habla David Ugarte. Se trata de una organización no gubernamental, sin afiliación política o religiosa dedicada a conectar a jóvenes con la clase política mediante el impulso de iniciativas que permiten el acercamiento de líderes políticos a la juventud. La organización utiliza su infraestructura y contactos para impulsar las inquietudes de la juventud para que ésta tenga una voz en el proceso político. Generation Engage está basada en tres premisas fundamentales: los jóvenes carecen de acceso a la política (no es un problema de falta de interés), la democracia debe ser un diálogo y no un monólogo, y la mejor inversión en el futuro de una democracia es en jóvenes líderes a nivel local. Como embajador/voluntario de esta organización trabaja en la capital de Carolina del Norte, el español Jorge Piernavieja Nomdedeu quien formó parte del 'ejército' que movilizó al electorado joven del estado: "No es ningún secreto que la inmensa mayoría de los jóvenes que han votado, incluso muchos por primera vez, lo hicieron a favor de Obama. El número de voluntarios con los que contó el Partido Demócrata fue abrumador, debido a esta nueva forma de hacer política que involucra a la gente desde la base. Obama pasó diez veces por Carolina del Norte durante la campaña presidencial y en cada discurso finalizaba con el mismo mensaje: 'Os pido que habléis con vuestros vecinos y amigos y que les habléis del mensaje que estamos tratando de hacer llegar a todos los rincones del país'. El llamar de puerta en puerta, cubriendo vecindarios, es una práctica cada vez más común en los Estados Unidos, y el equipo de Obama la empleó hasta límites insospechados y nosotros, de alguna manera, formamos parte de ese debate ciudadano cara a cara al acercar la política a los universitarios" ${ }^{14}$.

Generation Engage trabaja redes reales y virtuales, Jorge Piernavieja considera que hay un antes y un después en la utilización del ciberactivismo y la Política 2.0 por parte de Obama. "Sus asesores pasarán a la historia como los mejores de la política norteamericana. Desde un principio supieron valorar el potencial del senador de Illinois y forjar un mito en Internet. La juventud no toca un periódico, mis compañeros de Universidad descubrieron a Obama en Internet, en You Tube, en

\footnotetext{
${ }^{12}$ González, J.L., 'Modelos de periodismo local y estrategias ante la crisis: el caso del News \& Observer', Revista Latina de Comunicación Social 64, enero de 2009, La Laguna (Tenerife), pgs151-160

${ }_{13}$ Ugarte, D, El poder de las redes, Ed. Sociedad de las Indias Electrónicas, Madrid, 2008

14 Piernavieja Nomdedeu, J, entrevista personal con el embajador-voluntario de Generation Engage en Carolina del Norte, Raleigh (EE.UU), noviembre de 2008
} 
las redes sociales, fundamentalmente My Space y Facebook, en Estados Unidos. Sin lugar a dudas, las campañas se disputan ya en la Red, y el logro de la campaña de Obama fue saber poner el mensaje adecuado en la Red, esperar que la gente respondiera y dialogar con ella". Buen ejemplo de todo ello es que Generation Engage, muy asentada en Carolina del Norte, concentra su ciberactividad en la Red, como no podía ser de otra manera, y desde la Red han conseguido aglutinar el interés de muchos jóvenes universitarios del estado por la política real.

\subsection{El activismo 'puerta a puerta' de 'Moms for Obama'}

Otro ejemplo de activismo ciudadano en pos del mismo fin: conseguir la victoria de Obama, es el de la organización Moms for Obama. Diferente, en su estrategia y estructura, a la organización de Generation Engage, Moms for Obama es el ejemplo perfecto de pequeñas redes vecinales que van creciendo exponencialmente hasta aglutinar a miles de mujeres que votaron por Obama. Sara Cork dirigió una pequeña 'célula' de Moms for Obama en Chapel Hill (Carolina del Norte). Su misión durante un año (2008) fue organizar todos los sábados pequeñas reuniones en su casa, con no más de diez mujeres, para hablar de política, fundamentalmente local, para desglosar los programas de Beverly Perdeu y de la senadora Key Hagan y, por supuesto, para hablar de la necesidad de cambiar el color político de la Casa Blanca. El único anuncio de dichas reuniones era un cartel en la puerta de la casa, con la hora prevista. "Moms for Obama fue un movimiento espontáneo de mujeres demócratas que considerábamos fundamental llevar a nuestros vecinos la idea del cambio. Las reuniones daban lugar a otras reuniones en otras casas y así sucesivamente por todo el estado, recaudándose fondos para la campaña demócrata, organizándose rifas, cenas benéficas y otras acciones solidarias y de concienciación política" ${ }^{15}$. En este caso la estrategia en la Red era de mero apoyo, aunque el objetivo final era llegar un perfil de la población que podía resultar decisivo: las mujeres, y dentro de las mujeres, las mujeres mayores de raza negra. Como bien apuntaba Javier Piernavieja, "nunca la raza fue tan importante en unas elecciones norteamericanas".

Finalmente, con el objeto de culminar la presente investigación se preguntó a 125 universitarios de NCSU, con edades comprendidas entre los 18 y los 53 años, y de distintas razas y condición social, sobre como habían influido las redes sociales reales, las virtuales y los medios de comunicación en su voto. Un $51 \%$ de los encuestados aseguró que alguno de sus progenitores había participado en reuniones, charlas informativas 0 actividades de asesoramiento 0 propaganda. Un $38 \%$ de los encuestados destacó que su madre había participado directamente 0 indirectamente en las actividades de Moms for Obama. Hasta un 65\% dijo conocer Generation Engage, bajando hasta un $45 \%$ los que habían participado en algunas de las sesiones 0 actividades organizadas por esta organización. El 90\% de los estudiantes encuestados siguió la campaña a través de redes sociales, fundamentalmente My Space y Facebook, por este orden, y a través de medios online. Solamente un $8 \%$ afirmó seguir la campaña a través de la prensa escrita, por un $50 \%$ que dijo seguir los debates televisivos entre los candidatos. Preguntados sobre su voto el 4 de noviembre de 2008 en las elecciones presidenciales norteamericanas, de los 125 encuestados, 114 se registraron para votar y votaron: Obama (68), McCain (43), Bob Barr (1), NS/NC (2).

\footnotetext{
${ }^{15}$ Cork, S, entrevista personal con la responsable de Moms for Obama en Chapel Hill. Chapel Hill (EE.UU), noviembre 2008
} 


\section{Conclusiones}

Tras un año de la llegada de Obama a la Casa Blanca y del vuelco electoral que se produjo en Carolina del Norte, se puede comprobar que la popularidad del presidente norteamericano sigue siendo muy alta, a pesar de la crisis internacional que está azotando a Estados Unidos de manera significativa. Tal circunstancia tiene que ver con que Obama no ha cometido ningún error grave en su mandato durante estos meses, está cumpliendo su programa electoral, siendo especialmente positiva la trayectoria internacional y, finalmente, ha consiguió tejer una red de apoyo ciudadano durante la campaña que es su mejor salvavidas para tiempos de crisis y será su mejor valor para una posible reelección. El periodista Ignacio Ramonet resume una de las claves del éxito de Obama, tanto para llegar a la Casa Blanca como para mantener esa popularidad un año después: "Transmitir un sentimiento de confianza, el de un hombre que mantiene su serenidad a pesar de las presiones y que no se deja desestabilizar"16.

En conclusión, el Partido Demócrata, con Obama a la cabeza, consiguieron un vuelco político sin precedentes en Carolina del Norte, gracias al apoyo ciudadano fraguado 'puerta a puerta' sustentado en el trabajo de los 27.000 voluntarios del propio partido, como de otras organizaciones independientes progresistas como Generation Engage o Moms for Obama que hicieron didáctica electoral en un momento político clave para el estado y para el conjunto del país. Esa didáctica política en zonas urbanas universitarias resultó clave para recuperar y activar un voto joven que respaldó a Obama, Perdue y Hagan. Ese voto joven encontró la puerta de la política a través de la Política 2.0: sitios online, redes sociales y blogs. La raza fue un factor determinante en estas elecciones, en Carolina del Norte el voto negro fue mayoritariamente para Obama, también el latino, en una zona del país donde el perfil poblacional está cambiando a pasos agigantados. En definitiva, la simbiosis entre redes sociales reales y virtuales, destinadas a construir un sólido diálogo con el ciudadano, fueron la clave del histórico vuelco electoral objeto de estudio en este artículo, un apoyo nada efímero que parece consolidado para mucho tiempo.

\section{BIBLIOGRAFÍA:}

Barkin, Dan, entrevista personal con el editor online del News \& Observer, Raleigh (EE.UU), noviembre 2008.

Editorial, 'Juventud y participación política', Fundación Sistema, Temas para Debate 176, julio de 2009, Madrid, pgs. 3-4.

Freire, J, 'Internet en la campaña electoral americana', Soitu, noviembre de 2008, Madrid, disponible en: http://www.soitu.es/soitu/2008/10/02/pieldigital/1222965797 622282.htm

Meek, Jerry, entrevista personal con el presidente del Partido Demócrata en Carolina del Norte, Raleigh (EE. UU), noviembre 2008.

Olivieri, Luis, entrevista personal con el politólogo de North Carolina State University, Raleigh (EE. UU), noviembre 2008.

Pastor Seller, E, 'Participación ciudadana y política social', Ed. Azacanes, Praxis 13, marzo de 2009, Toledo, pgs. 110-127

Quintas Froufe, Eva y Natalia (2010): "Cara a cara electoral televisado. Análisis audiovisual de los debates entre los candidatos a la Presidencia del Gobierno Española (2008)", en Miguel Hernández Communication Journal, 1, páginas 20 a 39. Universidad Miguel Hernández, UMH (Elche-Alicante). Recuperado el 10 de Enero de 2010 de: http://mhcj.es/2010/01/23/quintas-froufe.

\footnotetext{
${ }^{16}$ Ramonet, I, 'Obama, seis meses después', Le Monde Diplomatique 165, julio 2009, Valencia, pgs 1-2
} 
Varela, J, 'Con Obama en el iPhone', Soitu, noviembre de 2008, Madrid, disponible en: http://soitu.es/soitu/2008/10/04/sociedadcableada/1223105526 450020.html 